\title{
Optimization of the Expression Conditions of CGA-N46 in Bacillus subtilis DB1342(p-3N46) by Response Surface Methodology
}

\author{
Rui-Fang $\mathrm{Li}^{1} \cdot$ Bin Wang ${ }^{1} \cdot$ Shuai Liu ${ }^{1} \cdot$ Shi-Hua Chen ${ }^{1} \cdot$ Guang-Hai Yu ${ }^{1}$ • \\ Shuo-Ye Yang ${ }^{1} \cdot$ Liang Huang $^{1} \cdot$ Yan-Li Yin ${ }^{1} \cdot{\text { Zhi-Fang } \mathrm{Lu}^{1}}^{1}$
}

Received: 7 November 2014/Revised: 4 January 2015/Accepted: 30 January 2015/Published online: 4 September 2015

(c) The Author(s) 2015. This article is published with open access at Springerlink.com

\begin{abstract}
CGA-N46 is a small antifungal-derived peptide and consists of the 31st-76th amino acids of the N-terminus of human chromogranin A. Polycistronic expression of recombinant CGA-N46 in Bacillus subtilis DB1342 was used to improve its production, but the yield of CGA-N46 was still low. In the present study, response surface methodology (RSM) was used to optimize culture medium composition and growth conditions of the engineered strain B. subtilis DB1342(p-3N46) for the further increase in CGA-N46 yield. The results of two-level factorial experiments indicated that dextrin and tryptone were significant factors affecting CGA-N46 expression. Central composite design (CCD) was used to determine the ideal conditions of each significant factors. From the results of CCD, the optimal medium composition was predicted to be dextrin $16.6 \mathrm{~g} / \mathrm{L}$, tryptone $19.2 \mathrm{~g} / \mathrm{L}, \mathrm{KH}_{2} \mathrm{PO}_{4} \cdot \mathrm{H}_{2} \mathrm{O} 6 \mathrm{~g} / \mathrm{L}, \mathrm{pH}$ 6.5. And the optimal culture process indicated inoculation of $B$. subtilis DB1342(p-3N46) seed culture into fresh culture medium at $5 \%(\mathrm{v} / \mathrm{v})$, followed by expression of CGA-N46 for 56 hours at $30{ }^{\circ} \mathrm{C}$ induced by $2 \%(\mathrm{v} / \mathrm{v})$ sucrose after one hour of shaking culture. To test optimal CGA-N46 peptide expression, the yeast growth inhibition assay was employed and it was found that under optimal culture conditions, CGA-N46 inhibited the growth of Candida albican by $42.17,30.86 \%$ more than that in the pre-optimization conditions. In summary, RSM can be used to optimize expression conditions of CGA-N46 in engineered strains B. subtilis DB1342(p-3N46).
\end{abstract}

Rui-Fang Li

1375098398@qq.com

1 College of Biological Engineering, Henan University of Technology, Zhengzhou 450001, China
Keywords Bacillus subtilis engineered strain - Genetic engineering expression · CGA-N46 · Culture conditions optimization - Two-level factorial experiments - Response surface methodology - Biostatistic computer software . Biostatistic analysis · Antifungal peptide

\section{Introduction}

Candida albican, a ubiquitously distributed opportunistic pathogen, is the leading cause of candidas and causes one of the highest numbers of deaths among patients with fungal infections in the world [1, 2, 3]. Azole drugs are commonly used to treat infections caused by $C$. albican . However, some Candida spp. are intrinsically resistant or have reduced susceptibility to antifungal agents $[4,5,6]$. Antimicrobial peptides (AMPs) are multidimensionality powerful host shield and are hard for microorganisms to overcome using single approach resistance strategies. AMPs have shown to be effective alternatives to the current antimycotic therapies with the increasing resistance to conventional antimycotic drugs [7, 8]. CGA-N46, a peptide containing the 31 st -76 th amino acid of human chromogranin A, has antagonistic activity to C. albican [9]. To meet the need for drug development for future clinical applications, an abundance of CGA-N46 is necessary.

There are numerous approaches to optimizing recombinant expression. They are mainly two classes, i.e., genetic engineering and culture conditions optimization. The approaches on high efficient expression optimization mainly were genetic engineering, such as fusion expression $[10,11]$, induced expression $[12,13]$, or targeted codon optimization [14]. Culture conditions optimization were conducted in high-density cultivation [13, 15]. Li et al. [16] previously constructed an inducible tri-cistronic expression 
plasmid p-3N46 containing CGA-N46, which allowed three copies of $c g a-\mathrm{N} 46$ gene be induced to express in one plasmid. Using this genetic strain, the expression of $\mathrm{cga}$ N46 gene was increased by optimizing plasmid.

Production yield and cost of recombinant proteins are considerably influenced by bacterial culture conditions and medium composition. Optimization of culture conditions using biostatistic methods is another method to increase the expression of an engineered strain. Because of the difficulty of purification, one-factor-at-a-time was usually used [17]. However, this method frequently failed to locate the region of optimal response. Response surface methodology (RSM), exploring the relationships between several explanatory variables and one or more response variables [18], is characterized by more variables, fewer experiments, shorter cycle, and higher accuracy than other statistical optimization techniques such as one-factor-at-a-time $[19,17]$ or orthogonal tests. The main idea of RSM is to use a sequence of designed experiments to obtain an optimal response by using a second-degree polynomial model. As an optimization method, the experimental random error is considered in RSM, which is not in other traditional biostatistic methods. RSM is a simple, useful, and more precise methodology that can determine optimal culture medium composition and culture conditions using biostatistic computer software. RSM is commonly used for enhancing microorganism metabolite production at present [20,21, 22, 18]. However, it is rarely reported in engineered strain expression.

In this study, RSM was used to optimize the medium and culture conditions for maximal expression of CGAN46. To quantify CGA-N46, its effectiveness on inhibiting C. albican growth were tested.

\section{Methods}

\subsection{Microorganisms}

The engineered strains $B$. subtilis DB1342(pSBPTQ) which bore plasmid pSBPTQ [23] and B. subtilis DB1342(p3N46) which bore plasmid p-3N46 [17] used in this study were previously constructed.

Plasmid pSBPTQ, an Escherichia coli and Bacillus subtilis shuttle plasmid, was a cloning plasmid with ampicillin resistance $\left(A m p^{r}\right)$ in E. coli and an expression plasmid with kanamycin resistance $\left(\mathrm{Kan}^{r}\right)$ in $B$. subtilis. It had $s a c \mathrm{~B}$ promoter sequence ( $\operatorname{sac} \mathrm{B} p s$ ) which can be induced by sucrose and promote the transcription of the exogenous gene downstream of $s a c \mathrm{~B} p s$. It had multicloning sites between $s a c \mathrm{~B} p s$ and terminator sequence $(T)$ which allowed inserting exogenous gene in pSBPTQ. The terminator could make an efficient stop of the transcription. Plasmid p-3N46 was a recombinant vector from

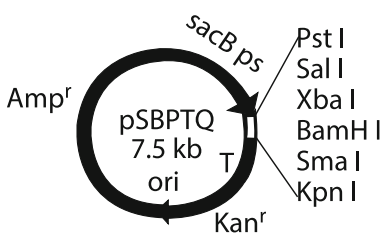

(a)

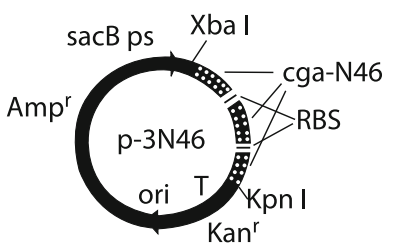

(b)
Fig. 1 Physical map of pSBPTQ (a) and p-3N46 (b)

pSBPTQ which bore three cistrons of cga-N46 gene between $s a c \mathrm{~B} p s$ and terminator in pSBPTQ. Each $c g a$ $\mathrm{N} 46$ cistron had one ribosome binding site, one initiating codon, one copy of cga-N46 gene, one stop codon. Three cistrons of cga-N46 gene allowed three copies of CGAN46 to be expressed in a single plasmid p-3N46. Their physical maps are shown in Fig. 1.

Yeast strain C. albican CSC314 was supplied by the Institute of Dermatology, Chinese Academy of Medical Sciences.

\subsection{Culture Media and Growth Conditions}

Luria-Bertani (LB) agar medium (10 $\mathrm{g}$ tryptone, $5 \mathrm{~g}$ yeast extract, $10 \mathrm{~g} \mathrm{NaCl}, 18 \mathrm{~g}$ agar in $1000 \mathrm{~mL}$ of distilled water, $\mathrm{pH}$ 7.0) containing $10 \mu \mathrm{g} / \mathrm{mL}$ kanamycin was used to culture B. subtilis DB1342 (pSBPTQ) and B. subtilis DB1342(p-3N46). The LB broth medium was prepared with the components as LB agar medium except agar as the growing culture for bacteria.

$2 \times$ MSR medium [24], contained glucose $10 \mathrm{~g}$, tryptone $30 \mathrm{~g}$, yeast extract $50 \mathrm{~g}, \mathrm{KH}_{2} \mathrm{PO}_{4} 6 \mathrm{~g}$ in $1000 \mathrm{~mL}$ of distilled water, was used for expression culture optimization studies.

YPD medium (yeast extract $10 \mathrm{~g}$, peptone $20 \mathrm{~g}$, dextrose $20 \mathrm{~g}$ in $1000 \mathrm{~mL}$ of distilled water) was used to culture $C$. albican CSC314.

The seed culture was prepared by transferring a loopful of fresh transformed strains B. subtilis DB1342(pSBPTQ) and B. subtilis DB1342(p-3N46) cells into $25 \mathrm{ml}$ LB broth in $250 \mathrm{~mL}$ Erlenmeyer flask. The flask was incubated at $250 \mathrm{rpm}$ at $37{ }^{\circ} \mathrm{C}$ for $16 \mathrm{~h}$.

\subsection{Expression Conditions}

The production process was carried out, unless otherwise mentioned, in Erlenmeyer flasks $(500 \mathrm{~mL})$ containing 100 $\mathrm{mL}$ aliquots of the basal culture medium with $10 \mu \mathrm{g} / \mathrm{mL}$ kanamycin. After cultivated for $2 \mathrm{~h}$ at $37{ }^{\circ} \mathrm{C} 200 \mathrm{rpm}, 5 \mathrm{~mL}$ $40 \%$ sterile sucrose solution was added into culture medium to induce the expression of CGA-N46 for $48 \mathrm{~h}$. During this period, CGA-N46 was expressed and secreted into culture supernatant. After expression, both of the 
culture were centrifuged at $12,000 \times g$ for $10 \mathrm{~min}$ at $4{ }^{\circ} \mathrm{C}$. For antagonistic activity determination, $10 \mathrm{~mL}$ culture supernatant was filtered with $0.22 \mu \mathrm{m}$ filter film.

\subsection{Assay of Antagonistic Activity}

To analyze the antagonistic activity, $10 \mathrm{~mL}$ supernatant was mixed with equal volume of sterile YPD medium. C. albican $\left(10^{6}\right.$ cells $\left./ \mathrm{mL}\right)$ was inoculated into the mixture medium at $10 \%(\mathrm{v} / \mathrm{v})$ and then incubated at $26^{\circ} \mathrm{C}, 200 \mathrm{rpm}$. The supernatant from B. subtilis DB1342(pSBPTQ) was used as control. After incubating for $24 \mathrm{~h}$, the yeast cells were centrifuged at $10,000 \times g$ for $10 \mathrm{~min}$ at room temperature. The cell precipitates were dried in a constant temperature oven at $37{ }^{\circ} \mathrm{C}$ to remove all traces of water. The dried cell precipitates were weighed, and yeast growth inhibition caused by CGA-N46 was calculated according to Eq. (1).

$Y=\left(W_{2}-W_{1}\right) / W_{2} \times 100 \%$

Here, $W_{1}$ was the yeast cell dry weight in experimental group, $W_{2}$ was the yeast cell dry weight in control group, and $Y$ was the indicator of yeast growth inhibition rate.

All experiments were conducted in triplicate, and the mean values were reported with standard error.

\subsection{Carbon Source Screening}

The carbon source in $2 \times$ MSR medium was replaced by glycerol, maltose, lactose, dextrin, and soluble starch, respectively, to optimize the carbon source of the culture medium. B. subtilis DB1342(p-3N46) seed culture was inoculated into the carbon source modified $2 \times$ MSR medium to express CGA-N46. Yeast growth inhibition rates of B. subtilis DB1342(p-3N46) culture supernatant from each medium were measured.

\subsection{Nitrogen Source Screening}

To study the effect of nitrogen source of the culture medium on the antagonistic activity of culture supernatant, the nitrogen source in $2 \times$ MSR medium was replaced by casein, peptone, $\mathrm{KNO}_{3}$, ammonium sulfate, and urea, respectively. B. subtilis DB1342(p-3N46) seed culture was inoculated into nitrogen source modified $2 \times$ MSR medium to express CGA-N46. Yeast growth inhibition rates of $B$. subtilis DB1342(p-3N46) culture supernatant from each medium were measured.

\subsection{Computer Software for Experimental Design and Statistical Analysis}

The computer software, Design Expert 7.1 (Stat-Ease, Inc, Minneapolis, MN), was used for experimental design and regression analysis of the experimental data. For analysis of the fitted response nature and prediction of the maximum point, second-order equation was reduced to its canonical form.

\subsection{Response Surface Methodology for Optimizing Culture Conditions}

\subsubsection{Two-Level Factorial Experiment}

Two-level factorial experiments were used to find significant factors. Considering the culture conditions reported in previous studies, the main factors in this study were set as follow (as shown in Table 1): dextrin $(A)$, tryptone $(B)$, initial medium $\mathrm{pH}(C)$, seed culture inoculation concentration $(D)$, sucrose added time $(E)$, induction period $(F)$, and induction temperature $(G)$. Design Expert 7.1 was used to design the experiments. Yeast growth inhibition rate $(Y)$ was designed as response values. The significant factors affecting the expression of CGA-N46 would be determined according to the response values of different experimental conditions. Each experiment was conducted in three replicates.

\subsubsection{The Steepest Descent Experiment}

In RSM, the optimal area of single significant factors could be further explored by designing the steepest descent experiments based on the results of two-level factorial experiments. It is also why RSM has higher accuracy than other statistical optimization techniques. These experimental designs are shown in Table 3. The optimal area of significant factors would be determined based on the response values.

\subsubsection{Central Composite Design}

In RSM, after the steepest ascent path experiments of single significant factors, the optimal level of significant factors would be further studied by central composite experiments. Central composite experiment design is shown in Table 4.

\section{Results and Discussion}

\subsection{Effect of Carbon Source on Yeast Growth Inhibition Rate}

Yeast growth inhibition rates of B. subtilis DB1342(p3N46) culture supernatant from each carbon source modified $2 \times$ MSR media were calculated, and the results are presented in Fig. 2. The maximum yeast growth inhibition 
Table 1 Two-level factorial experiment design and results

\begin{tabular}{llllllllr}
\hline Run & $A(\mathrm{~g} / \mathrm{L})$ & $B(\mathrm{~g} / \mathrm{L})$ & \multicolumn{1}{l}{$C$} & $D(\%)$ & $E(\mathrm{~h})$ & $F(\mathrm{~h})$ & $\mathrm{G}\left({ }^{\circ} \mathrm{C}\right)$ & \multicolumn{1}{l}{$Y(\%)$} \\
\hline 1 & $-1(10)$ & $-1(10)$ & $-1(6.5)$ & $-1(5)$ & $-1(1)$ & $-1(48)$ & $-1(30)$ & $42.24 \pm 0.77$ \\
2 & $+1(40)$ & $-1(10)$ & $-1(6.5)$ & $-1(5)$ & $-1(1)$ & $+1(56)$ & $+1(37)$ & $18.31 \pm 0.41$ \\
3 & $-1(10)$ & $+1(40)$ & $-1(6.5)$ & $-1(5)$ & $+1(2)$ & $+1(56)$ & $-1(30)$ & $16.8 \pm 0.56$ \\
4 & $+1(40)$ & $+1(40)$ & $-1(6.5)$ & $-1(5)$ & $+1(2)$ & $-1(48)$ & $+1(37)$ & $-7.82 \pm 2.04$ \\
5 & $-1(10)$ & $-1(10)$ & $-1(6.5)$ & $+1(10)$ & $+1(2)$ & $+1(56)$ & $+1(37)$ & $34.75 \pm 1.12$ \\
6 & $+1(40)$ & $-1(10)$ & $-1(6.5)$ & $+1(10)$ & $+1(2)$ & $-1(48)$ & $-1(30)$ & $15.3 \pm 0.38$ \\
7 & $-1(10)$ & $+1(40)$ & $-1(6.5)$ & $+1(10)$ & $-1(1)$ & $-1(48)$ & $+1(37)$ & $19.07 \pm 0.25$ \\
8 & $+1(40)$ & $+1(40)$ & $-1(6.5)$ & $+1(10)$ & $-1(1)$ & $+1(56)$ & $-1(30)$ & $6.7 \pm 0.44$ \\
9 & $-1(10)$ & $-1(10)$ & $+1(7.5)$ & $-1(5)$ & $+1(2)$ & $-1(48)$ & $+1(37)$ & $27.27 \pm 0.73$ \\
10 & $+1(40)$ & $-1(10)$ & $+1(7.5)$ & $-1(5)$ & $+1(2)$ & $+1(56)$ & $-1(30)$ & $22.38 \pm 0.40$ \\
11 & $-1(10)$ & $+1(40)$ & $+1(7.5)$ & $-1(5)$ & $-1(1)$ & $+1(56)$ & $+1(37)$ & $18.84 \pm 0.21$ \\
12 & $+1(40)$ & $+1(40)$ & $+1(7.5)$ & $-1(5)$ & $-1(1)$ & $-1(48)$ & $-1(30)$ & $-0.98 \pm 0.89$ \\
13 & $-1(10)$ & $-1(10)$ & $+1(7.5)$ & $+1(10)$ & $-1(1)$ & $+1(56)$ & $-1(30)$ & $31.26 \pm 0.74$ \\
14 & $+1(40)$ & $-1(10)$ & $+1(7.5)$ & $+1(10)$ & $-1(1)$ & $-1(48)$ & $+1(37)$ & $13.08 \pm 3.99$ \\
15 & $-1(10)$ & $+1(40)$ & $+1(7.5)$ & $+1(10)$ & $+1(2)$ & $-1(48)$ & $-1(30)$ & $15.98 \pm 0.46$ \\
16 & $+1(40)$ & $+1(40)$ & $+1(7.5)$ & $+1(10)$ & $+1(2)$ & $+1(56)$ & $+1(37)$ & $-6.6 \pm 3.83$ \\
17 & $0(25)$ & $0(25)$ & $0(7.0)$ & $0(7.5)$ & $0(1.5)$ & $0(52)$ & $0(33)$ & $17.42 \pm 0.15$ \\
18 & $0(25)$ & $0(25)$ & $0(7.0)$ & $0(7.5)$ & $0(1.5)$ & $0(52)$ & $0(33)$ & $17.07 \pm 0.74$ \\
19 & $0(25)$ & $0(25)$ & $0(7.0)$ & $0(7.5)$ & $0(1.5)$ & $0(52)$ & $0(33)$ & $23.37 \pm 0.02$ \\
20 & $0(25)$ & $0(25)$ & $0(7.0)$ & $0(7.5)$ & $0(1.5)$ & $0(52)$ & $0(33)$ & $19.3 \pm 0.26$ \\
\hline$A$ & & +10.53
\end{tabular}

$A$-dextrin, $B$-tryptone, $C$-medium $\mathrm{pH}, D$-seed culture inoculation concentration, $E$-sucrose added time, $F$-induction period, $G$-induction temperature, $Y$-yeast growth inhibition rate rate was $12.23 \%$ in dextrin modified $2 \times$ MSR medium, which increased $1.59 \%$ than that of the control (10.64\%). Therefore, dextrin was the optimal carbon source for CGAN46 expression.

\subsection{Effect of Nitrogen Source on Yeast Growth Inhibition Rate}

Yeast growth inhibition rates of B. subtilis DB1342(p3N46) culture supernatant from each nitrogen source

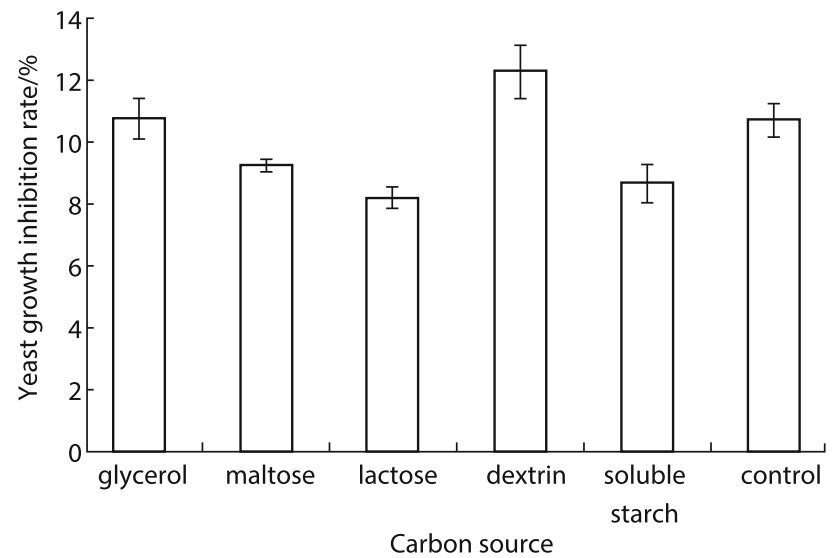

Fig. 2 Effect of different carbon source in modified $2 \times$ MSR medium on yeast growth inhibition rate of B. subtilis DB1342 (p3N46) culture supernatant modified $2 \times$ MSR media were calculated, and the results are shown in Fig. 3. The maximum yeast growth inhibition rate was $11.07 \%$ with tryptone as nitrogen resource (the control). Tryptone was chosen to be the optimal nitrogen source for CGA-N46 expression.

\subsection{Yeast Growth Inhibition Rate in Pre- optimization Conditions}

From the results of one-factor-at-a-time method for screening carbon and nitrogen sources, the culture medium composition was obtained as follows: yeast extract $50 \mathrm{~g} / \mathrm{L}$,

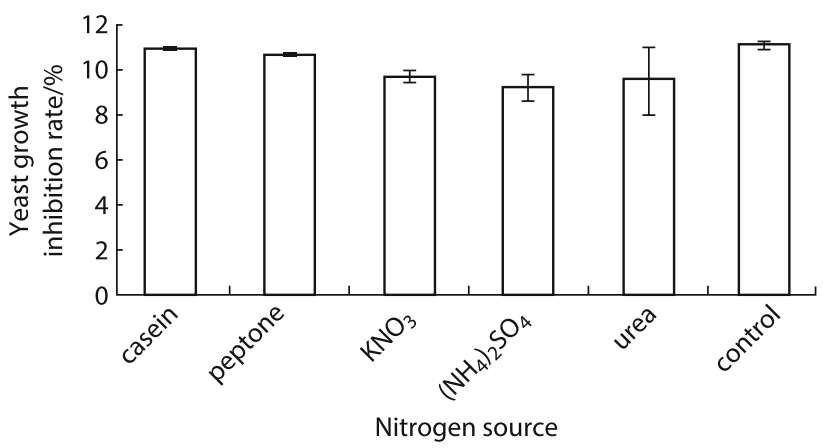

Fig. 3 Effect of different nitrogen source in modified $2 \times$ MSR medium on yeast growth inhibition rate of B. subtilis DB1342(p$3 \mathrm{~N} 46$ ) culture supernatant 
dextrin $10 \mathrm{~g} / \mathrm{L}$, tryptone $30 \mathrm{~g} / \mathrm{L}, \mathrm{KH}_{2} \mathrm{PO}_{4} \cdot \mathrm{H}_{2} \mathrm{O} 6 \mathrm{~g} / \mathrm{L}$. This culture medium was considered as pre-optimization medium. The antagonistic activity of B. subtilis DB1342(p3 N46) culture supernatant in pre-optimization conditions was calculated to be $11.31 \%$.

\subsection{Significant Factors}

There were 20 different experiments designed by software Design Expert 7.1 for two-level factorial experiment. The experiment results (i.e., response values) are shown in Table 1. The main effect analysis of the influencing factors is shown in Table 2. Dextrin and tryptone were two statistically significant factors $(p<0.05)$ that affected the yeast growth inhibition rate of B. subtilis DB1342(p-3N46) culture supernatant.

The multiple regression equation of the response values (yeast growth inhibition rate) for the independent variables was obtained by software analysis.

$$
\begin{aligned}
Y= & 13.94-8.09 A-9.44 B-1.03 C-0.83 D \\
& -1.26 E+1.21 F-3.19 G
\end{aligned}
$$

In Eq. (2), the independent variables included dextrin $(A)$, tryptone $(B)$, medium $\mathrm{pH}(C)$, seed culture inoculation concentration $(D)$, sucrose added time $(E)$, induction period $(F)$, and induction temperature $(G)$. From the coefficients of different variables in Eq. (2), the statistically nonsignificant factors, including medium $\mathrm{pH}$, seed and induction temperature were negative factors for response value, while the statistically nonsignificant factor induction period was positive one. Therefore, the -1 level for negative factors and the +1 level for positive factor were chosen for subsequent experiments. The values of the statistically nonsignificant factors were as follows: $\mathrm{pH}$ 6.5, the seed culture inoculation concentration $5 \%(\mathrm{v} / \mathrm{v})$, sucrose added after the seed culture inoculated for $1 \mathrm{~h}$, induction temperature $30^{\circ} \mathrm{C}$, and induction period $56 \mathrm{~h}$. culture inoculation concentration, sucrose added time,

\subsection{The Steepest Descent Path Test Results of Significant Factors}

The coefficients of significant factors dextrin $(A)$ and tryptone $(B)$ in Eq. (2) suggested that reducing the concentrations of dextrin and tryptone could improve the response value (yeast growth inhibition rate). The steepest descent path tests of single factor were designed to further optimize the significant factors. As initiation concentration of the descent path, $40 \mathrm{~g} / \mathrm{L}$ dextrin and tryptone were chosen , and 5 $\mathrm{g} / \mathrm{L}$ was set as the step length. The results in Table 3 showed that the response value of $B$. subtilis DB1342(p-3N46) supernatant in run 6 (dextrin $15 \mathrm{~g} / \mathrm{L}$ and tryptone $15 \mathrm{~g} / \mathrm{L}$ ) was $41.11 \%$, suggesting that the optimal concentrations of dextrin and tryptone for response value were near $15 \mathrm{~g} / \mathrm{L}$.

\subsection{Central Composite Experimental Results}

To get the exact optimal concentrations of significant factors, central composite experiments were performed. $15 \mathrm{~g} / \mathrm{L}$ dextrin and $15 \mathrm{~g} / \mathrm{L}$ tryptone were set as the central value. The levels of dextrin and tryptone and central composite experiment designs are shown in Table 4. A regression equation was obtained for response value when RSM analysis was used to evaluate dextrin and tryptone variables.

$Y=41.13-0.63 A+7.07 B+9.24 A B-7.63 A^{2}-4.68 B^{2}$

Table 3 Steepest ascent experiment design and results

\begin{tabular}{lllr}
\hline Run & $A(\mathrm{~g} / \mathrm{L})$ & $B(\mathrm{~g} / \mathrm{L})$ & \multicolumn{1}{c}{$\mathrm{Y}(\%)$} \\
\hline 1 & 40 & 40 & $9.06 \pm 0.24$ \\
2 & 35 & 35 & $12.73 \pm 2.00$ \\
3 & 30 & 30 & $9.73 \pm 1.02$ \\
4 & 25 & 25 & $30.877 \pm 1.88$ \\
5 & 20 & 20 & $36.90 \pm 1.48$ \\
6 & 15 & 15 & $41.11 \pm 1.87$ \\
7 & 10 & 10 & $34.76 \pm 2.50$ \\
8 & 5 & 5 & $33.16 \pm 2.29$ \\
\hline$A-$ dextrin, $B-$ tryptone, $Y$ - yeast growth inhibition rate
\end{tabular}

Table 2 Main effect analysis of two-level factorial experiment

\begin{tabular}{lcccccc}
\hline Source & Sum of squares & $d f$ & Mean square & $F$ value & $p$ value $($ Prob $>F)$ & Significance \\
\hline$A$ & 1047.66 & 1 & 1047.66 & 24.89 & 0.0004 & $* * *$ \\
$B$ & 1426.76 & 1 & 1426.76 & 33.90 & 0.0001 & $* * *$ \\
$C$ & 17.04 & 1 & 17.04 & 0.40 & 0.5376 & \\
$D$ & 11.04 & 1 & 11.04 & 0.261 & 0.6187 & \\
$E$ & 23.60 & 1 & 23.60 & 0.56 & 0.4697 & \\
$F$ & 25.38 & 1 & 25.38 & 0.60 & 0.4538 & \\
$G$ & 162.37 & 1 & 162.37 & 3.86 & 0.0753 & \\
\hline
\end{tabular}

*** indicate most significant values 
Table 4 Central composite experiment design and results

\begin{tabular}{lccr}
\hline Run & $A(\mathrm{~g} / \mathrm{L})$ & $B(\mathrm{~g} / \mathrm{L})$ & $Y(\%)$ \\
\hline 1 & $-1(10)$ & $+1(20)$ & $34.78 \pm 2.18$ \\
2 & $+1(20)$ & $-1(10)$ & $14.93 \pm 1.20$ \\
3 & $-1(10)$ & $-1(10)$ & $25.12 \pm 1.05$ \\
4 & $+1(20)$ & $+1(20)$ & $36.52 \pm 0.98$ \\
5 & $-\alpha(7.9)$ & $0(15)$ & $26.3 \pm 0.72$ \\
6 & $+\alpha(22.1)$ & $0(15)$ & $24.55 \pm 1.50$ \\
7 & $0(15)$ & $-\alpha(7.9)$ & $17.61 \pm 0.82$ \\
8 & $0(15)$ & $+\alpha(22.1)$ & $45.05 \pm 1.18$ \\
9 & $0(15)$ & $0(15)$ & $41.03 \pm 0.43$ \\
10 & $0(15)$ & $0(15)$ & $41.54 \pm 0.45$ \\
11 & $0(15)$ & $0(15)$ & $41.76 \pm 0.42$ \\
12 & $0(15)$ & $0(15)$ & $41.75 \pm 0.38$ \\
\hline
\end{tabular}

$A$-Dextrin, $B$-Tryptone, $Y$-Yeast growth inhibition rate

The variance of regression equation was analyzed by software Design Expert 7.10. The results are shown in Table 5 and Fig. 4.

The interaction effect of significant variables (dextrin and tryptone) on the response value (yeast growth inhibition rate) was studied by plotting three-dimensional surface curve. The three-dimensional curve of the calculated response (yeast growth inhibition rate) and contour plot from the interaction between the variables are shown in Fig. 4. The optimal concentration of dextrin and tryptone were 16.6 and $19.2 \mathrm{~g} / \mathrm{L}$, respectively. The theoretical maximum value of the yeast growth inhibition rate of DB1342(p-3N46) culture supernatant was $43.98 \%$.

\subsection{Validation of Experimental Model}

Bacillus subtilis DB1342(p-3N46) and DB1342(pSBPTQ) were cultured under the optimized conditions. The yeast growth inhibition rate of $B$. subtilis DB1342(p-3N46) supernatant was $42.17 \%$, which was very close to the theoretical result $43.98 \%$. The result suggested that the experimental model was quite accurate. The results also showed that the inhibition rate of DB1342(p-3N46)

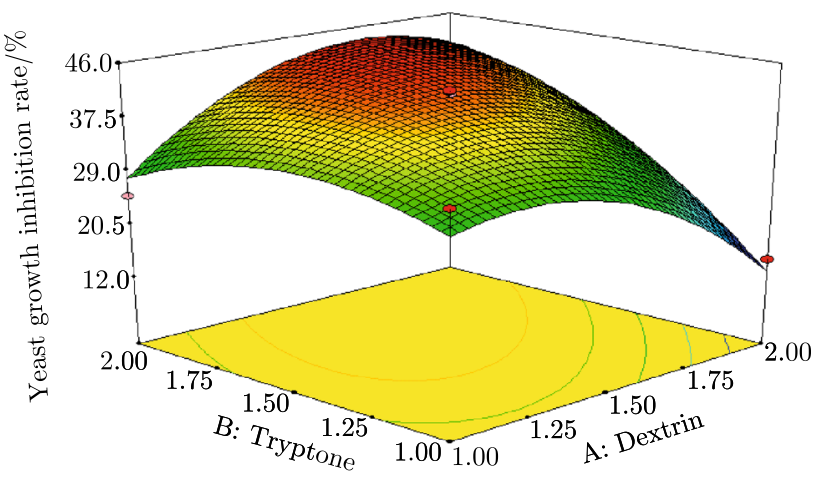

Fig. 4 Three-dimensional surface curve showing the effect of dextrin and tryptone interaction on yeast growth inhibition rate

supernatant from optimal conditions was significantly increased compared to the original conditions.

Compared with genetic engineering, the enhanced yield of CGA-N46 in culture conditions optimization was less, but it could be a valuable compensation to genetic engineering. Moreover, culture conditions optimization is a method to reduce the cost of expression for CGA-N46 industrial development.

This paper focused on investigation of significant factors that were responsible for peptide CGA-N46 expression in B. subtilis engineered strain DB1342(p-3N46) using mathematical and computational methods. The factors, such as nitrogen source and carbon source of medium components, initial medium $\mathrm{pH}$, inoculum size, sucrose added time, and induction period, were considered. Although more factors were considered in this investigation than in other reports $[25,26]$, some other factors, such as metallic ions, could further improve engineered strain's expression. But for the facilitation of the purification, the medium needs to be as simple as possible. Therefore, only some basal culture factors were chosen as the variables in the present investigation.

\section{Conclusion}

Response surface methodology (RSM) experimental designs offered an efficient and feasible approach for culture conditions optimization of CGA-N46 expression in B. subtilis

Table 5 AVONA

\begin{tabular}{lccccl}
\hline Source & Sum of squares & $d f$ & Mean square & $F$ value & $p$ value $($ Prob $>F)$ \\
\hline Model & 1183.82 & 5 & 1183.82 & 24.79 & 0.0006 \\
$A$ & 3.22 & 1 & 3.22 & 0.34 & 0.5866 \\
$B$ & 400.25 & 1 & 400.25 & 0.03 & 0.0006 \\
$A B$ & 341.14 & 1 & 341.14 & 35.37 & 0.0010 \\
$A^{2}$ & 372.89 & 1 & 372.89 & 0.99 & 0.0008 \\
$B^{2}$ & 140.21 & 1 & 140.21 & 0.53 & 0.0086 \\
\hline
\end{tabular}


DB1342(p-3N46). The optimal medium composition was yeast $50 \mathrm{~g} / \mathrm{L}$, dextrin $16.6 \mathrm{~g} / \mathrm{L}$, tryptone $19.2 \mathrm{~g} / \mathrm{L}, \mathrm{KH}_{2} \mathrm{PO}_{4-}$ $\mathrm{H}_{2} \mathrm{O} 6 \mathrm{~g} / \mathrm{L}, \mathrm{pH} 6.5$. The optimal culture conditions were inoculating seed culture at concentration of $5 \%(\mathrm{v} / \mathrm{v})$, adding sucrose up to $2 \%$ one hour after the seed culture was inoculated, and allowing cultures to incubate at $30{ }^{\circ} \mathrm{C}$ for 56 h. Under optimal culture conditions, yeast growth inhibition rate of CGA-N46 was $31.53 \%$ higher than that in original conditions. It was concluded that RSM was a method for efficiently improving the production of CGA-N46 in the engineered strain B. subtilis DB1342(p-3N46) and might provide an alternative approach to enhance the recombinant protein productivity in other engineered strains.

Acknowledgments This work was supported by the National Science Foundation of China (31071922) and grants from Science and Technology Plan Project of Henan Province (112102310325) and Henan University of Technology (11JCYJ10).

\section{Compliance with Ethical Standards}

Conflict of interest All authors declare no competing financial interests in the findings of this study.

Open Access This article is distributed under the terms of the Creative Commons Attribution License which permits any use, distribution, and reproduction in any medium, provided the original author(s) and the source are credited.

\section{References}

1. Brown GD, Denning DW, Gow NA, Levitz SM, Netea MG, White T (2012) Hidden killers: human fungal infections. Sci Transl Med 4:165rv13

2. Pfaller MA, Diekema DJ (2007) Epidemiology of invasive candidiasis: a persistent public health problem. Clin Microbiol Rev 20:133-163

3. Pfaller MA, Diekema DJ (2010) Epidemiology of invasive mycoses in North America. Crit Rev Microbiol 36:1-53

4. Bassetti M, Taramasso L, Nicco E, Molinari MP, Mussap M, Viscoli C (2011) Epidemiology, species distribution, antifungal susceptibility and outcome of nosocomial candidemia in a tertiary care hospital in Italy. PLoS one 6:e24198

5. Leroy O, Gangneux JP, Montravers P, Mira JP, Gouin F, Sollet JP, Carlet J, Reynes J, Rosenheim M, Regnier B, Lortholary O, Amar Cand Study Group (2009) Epidemiology, management, and risk factors for death of invasive Candida infections in critical care: a multicenter, prospective, observational study in France (2005-2006). Crit Care Med 37:1612-1618

6. Scorzoni L, de Lucas MP, Mesa-Arango AC, Fusco-Almeida AM, Lozano E, Cuenca-Estrella M, Mendes-Giannini MJ, Zaragoza O (2013) Antifungal efficacy during Candida krusei infection in non-conventional models correlates with the yeast in vitro susceptibility profile. PLoS one 8:e60047

7. Foubister V (2003) Superpeptide to treat Candida albicans. Drug Discov Today 8:380-381

8. Silva PM, Gonçalves S, Santos NC (2014) Defensins: antifungal lessons from eukaryotes. Frontiers Microbiol 5:1

9. Li R, Zhang T, Luo J (2006) Construction of an inducible and efficient expression secretion shuttle vector of B. subtilis. Acta Microbiol Sin 46:714-719 (in Chinese)
10. Wu D, Lu Y, Huang H, Ma L, Che Y, Zha X, Yao B, Yang P (2013) High-level secretory expression of metchnikowin in Escherichia coli. Protein Expres Purif 91:49-53

11. Xie YG, Han FF, Luan C, Zhang HW, Feng J, Choi YJ, Groleau D, Wang YZ (2013) High-yield soluble expression and simple purification of the antimicrobial peptide OG2 using the intein system in Escherichia coli. BioMed Res Int. doi:10.1155/2013/ 754319

12. Fan L, Kadura I, Krebs LE, Larson JL, Bowden DM, Frye CC (2013) Development of a highly-efficient CHO cell line generation system with engineered SV40E promoter. J Biotechnol 168:652-658

13. Nie Y, Yan W, Xu Y, Chen WB, Mu XQ, Wang X, Xiao R (2013) High-level expression of Bacillus naganoensis pullulanase from recombinant Escherichia coli with auto-induction: effect of lac operator. PLoS one 8:e78416

14. Hutterer KM, Zhang Z, Michaels ML, Belouski E, Hong RW, Shah B, Berge M, Barkhordarian H, Le E, Smith S, Winters D, Abroson F, Hecht R, Liu J (2012) Targeted codon optimization improves translational fidelity for an Fc fusion protein. Biotechnol Bioeng 109:2770-2777

15. Peng Z, Wang A, Feng Q, Wang Z, Ivanova IV, He X, Zhang B, Song W (2014) High-level expression, purification and characterization of porcine $\beta$-defensin 2 in Pichia pastoris and its potential as a cost-efficient growth promoter in porcine feed. Appl Microbiol Biotechnol 98:5487-5497

16. Li R, Wang B, Yi Y, Huang L, Xiong Q (2013) Polycistronic expression of CGA-N46 gene in Bacillus subtilis DB1342. Afr J Microbiol Res 7:3294-3303

17. Zarschler K, Witecy S, Kapplusch F, Foerster C, Stephan H (2013) High-yield production of functional soluble single-domain antibodies in the cytoplasm of Escherichia coli. Microb Cell Fact 12:97

18. Zhao X, Han Y, Tan X, Wang J, Zhou Z (2014) Optimization of antifungal lipopeptide production from Bacillus sp. BH072 by response surface methodology. J Microbiol 52:324-332

19. Givry S, Duchiron F (2008) Optimization of culture medium and growth conditions for production of L-arabinose isomerase and D-xylose isomerase by Lactobacillus bifermentans. Microbiology 77:281-287

20. He S, Wang H, Wu B, Zhou H, Zhu P, Yang R, Yan X (2013) Response surface methodology optimization of fermentation conditions for rapid and efficient accumulation of macrolactin A by marine Bacillus amyloliquefaciens ESB-2. Molecules 18:408-417

21. Li R, Xu Y (2011) Fermentation optimization to improve the production of antagonistic metabolites by Bacillus subtilis strain BS501a. J Cent South Univ Technol 18:1047-1053

22. Su X, Liu Y, Hu J, Ding L, Shen C (2014) Optimization of protein production by Micrococcus luteus for exploring pollutantdegrading uncultured bacteria. SpringerPlus 3:117

23. Li R, Zhang T, Luo J, Wang F, Gu Q, Gan J, Xiao F (2006) Antifungal activity fragments of $\mathrm{N}$ domain of chromogranin $\mathrm{A}$. Acta Sci Nat Univ Sunyatseni 45:64-67 (in Chinese)

24. Ye RQ, Kim JH, Kim BG, Szarka S, Sihota E, Wong SL (1999) High-level secretory production of intact, biologically active staphylokinase from Bacillus subtilis. Biotech Bioeng 62:87-96

25. Demir T, Gübe Ö, Yücel M, Hameş-Kocabaş EE (2013) Increased alkalotolerant and thermostable ribonuclease (RNase) production from alkaliphilic Streptomyces sp. M49-1 by optimizing the growth conditions using response surface methodology. World J Microbiol Biotechnol 29:1625-1633

26. Park K, Reardon KF (1996) Medium optimization for recombinant protein production by Bacillus subtilis. Biotech Lett 18:737-740 